\title{
Anatomie d'un système de guerre interne : le cas du Liban
}

Salim Nasr

\section{(2) OpenEdition}

1 Journals

\section{Édition électronique}

URL : http://journals.openedition.org/conflits/80

DOI : $10.4000 /$ conflits.80

ISSN : $1777-5345$

Éditeur :

CCLS - Centre d'études sur les conflits lilberté et sécurité, L'Harmattan

\section{Édition imprimée}

Date de publication : 21 janvier 1990

ISSN : 1157-996X

\section{Référence électronique}

Salim Nasr, «Anatomie d'un système de guerre interne : le cas du Liban », Cultures \& Conflits [En ligne], 01 | hiver 1990, mis en ligne le 30 décembre 2002, consulté le 30 mars 2021. URL : http://

journals.openedition.org/conflits/80 ; DOI : https://doi.org/10.4000/conflits.80

Ce document a été généré automatiquement le 30 mars 2021.

Creative Commons License 


\title{
Anatomie d'un système de guerre interne : le cas du Liban
}

\author{
Salim Nasr
}

1 La plupart des ouvrages et articles sur le conflit libanais ont plutôt porté sur son étiologie, ses origines, ses racines, ses antécédents, les facteurs qui ont contribué à son éclatement. Beaucoup moins (à l'exception d'approches de type journalistique ou descriptif) se sont intéressés au processus même du conflit, à la dynamique des facteurs et des forces qui ont constitué, au bout d'un certain nombre d'années, un système de guerre qui se reproduisait lui-même et qui avait généré sa propre sphère économique, ses propres strates sociales, une idéologie de la discorde qui justifiait la continuation du conflit, et des infrastructures économiques, politiques et militaires qui maintenaient l'état de guerre.

2 Le Liban d'aujourd'hui, après une demi génération de conflit violent, n'est pas essentiellement composé de communautés entières opposées les unes aux autres - les chrétiens, les musulmans, les chiites, etc. - ou même de fractions confessionnelles se combattant les unes les autres, ou même d'élites divisées ou de contre-élites qui luttent les unes contre les autres. Il y a évidemment un peu de chacune de ces contradictions. Mais, essentiellement, la situation actuelle, après quinze ans de guerre, reflète beaucoup plus l'existence d'un système de guerre cohérent, fonctionnel, qui domine une société civile en grande partie supprimée, fragmentée, asservie. Ce système de guerre qui fonctionne pour se reproduire à l'identique est manipulé par des patrons moyen-orientaux directement ou indirectement à travers leurs forces armées ou à travers leurs subventions et leurs envois d'armes.

Dès lors, le conflit libanais, comme plusieurs autres cas similaires, ne devrait pas être interprété comme une réalité quasi naturelle, comme une potentialité latente qui avait seulement besoin de conditions favorables pour être actualisée. On ne peut pas faire non plus comme si les communautés libanaises, à un moment historique déterminé, en 1975, s'étaient levées et avaient commencé à se combattre les unes les autres sur la base d'une hostilité cachée, profonde, nourrie par les préjugés et les mémoires collectifs. Le conflit libanais a été un long et douloureux processus d'éclatement délibéré des circuits 
économiques, culturels et interpersonnels; un processus organisé de déconstruction des habitudes de convivialité, d'arrangement et de tolérance.

4 Certes, il est évident que l'émergence d'un système de guerre au Liban implique une accumulation de tensions, de contradictions et de déséquilibres dans l'ordre social et politique de l'avant-guerre (donc, d'une certaine manière, la guerre est enfant de cette société) mais est-ce - et dans quelle mesure est-ce - un enfant légitime ou le produit d'un viol, même si des segments significatifs de cette société étaient consentants ou au moins complices? S'il demeure très important de comprendre les racines du conflit libanais, ses origines locales et extérieures, il est aussi important, sinon plus, de reconstituer l'émergence, la consolidation et la dénomination d'un système de guerre qui expliquent en grande partie son caractère prolongé, irrationnel et circulaire. Nous allons nous efforcer ici brièvement de présenter quelques-unes des caractéristiques de ce système de guerre comme système social, et de risquer une évaluation de ses possibilités de décomposition.

Les acteurs du système de guerre

5 Deux catégories majeures d'acteurs sont intervenues essentiellement dans le conflit au cours des quinze dernières années: les combattants libanais d'une part, et, d'autre part, les forces armées extérieures, c'est-à-dire non libanaises, qui sont devenues, à différents moments, des acteurs importants sur la scène libanaise. Globalement, au début des années 1990, on considère qu'il y a, toujours engagés sur la scène du conflit libanais, environ 12000 combattants. $55 \%$ d'entre eux représentent les différents éléments des forces armées extérieures au Liban, et $45 \%$ représentent les combattants libanais.

6 Les principales forces extérieures sont: les forces armées syriennes, 45000 hommes; les Palestiniens, les Iraniens et les Israéliens : les Palestiniens étaient peut-être 20000 au début de la guerre, mais leurs effectifs ont fluctué et ont connu leur point le plus bas en 1982 après l'invasion israélienne. Seulement, à la fin des années 1980, ils avaient largement reconstitué leurs forces armées qui sont estimées actuellement à plus de 15 000 combattants. Les 2000 à 3000 gardes révolutionnaires iraniens sont opérationnels depuis 1982-1983 dans la plaine de la Bekaa et dans certains secteurs du Sud-Liban. Quant aux 1500 à 2000 Israéliens, ils encadrent l'armée du Liban-Sud (2 500 combattants) et sont opérationnels dans la zone dite de sécurité qu'Israël a créée en 1976-1977, qu'elle a élargie en 1978, et qu'elle a de nouveau agrandie après l'invasion de 1982 et son retrait partiel du Sud-Liban en 1985. En tout, il y aurait plus de 65000 éléments armés appartenant aux différents acteurs régionaux.

Du côté des combattants libanais, on trouve d'abord l'armée libanaise, dont les effectifs ont, eux aussi, largement fluctué au cours du conflit. Ils ont atteint, à un certain moment, le chiffre de 30 000, mais sont descendus à moins de 8000 . Cette armée libanaise, dans ses différents bataillons et brigades, a suivi un développement parallèle aux grandes phases du conflit. Elle a connu trois grands éclatements: le premier au début de 1976; le deuxième en 1983-1984 après l'échec de la reconstitution de l'Etat sous le régime d'Amine Gemayel ; et le troisième en 1989-1990 après la non-élection présidentielle de l'été 1988, la constitution des deux gouvernements rivaux du général Aoun d'un côté et du Dr Hoss de l'autre. A chaque fois, l'État, ou ce qu'il en restait, a tenté de reconstruire l'armée nationale. La période actuelle voit de nouveau une tentative de ce genre avec la reconstruction de l'armée sous la direction du général Lahoud après la chute du général Aoun en octobre 1990. 
8 Ensuite, on trouve les milices. Il s'agit là évidemment d'une population beaucoup plus diversifiée et fragmentée. Plus d'une centaine de factions différentes sont apparues sur la scène depuis 1975 , une vingtaine d'entre elles ont joué un rôle significatif, à différentes périodes, et peut-être une dizaine ont survécu en ?990. Nous avons donc une sorte d'évolution darwinienne éliminant les factions les plus faibles, les plus petites ou les plus périphériques.

9 Les groupes les plus importants ayant survécu à ce type de "sélection" sont les suivants :

10 Recrutant essentiellement parmi la population chrétienne, tout en ayant des orientations politiques et des relations extérieures très différentes, il existe aujourd'hui quatre milices : les forces libanaises dirigées par Samir Geagea, et regroupant peut-être 8000 combattants ; à l'extrême sud, l'armée du Liban-Sud (2 500) dirigée par Antoine Lahad dans la zone de sécurité contrôlée par les Israéliens; le groupe des forces libanaises qui s'est séparé de la direction de Geagea en 1985 et qui est dirigé par Élie Hobeika. Expulsé des zones Beyrouth-Est en 1985, ce groupe de 2000 combattants était basé à Zahlé et dans la Bekaa ; il est récemment revenu dans la région de Metn après la chute ?u général Aoun. Enfin, à l'extrême nord du Liban, les Maradas, dirigés par le clan des Frangié, sont une milice essentiellement locale dans la région de Zgoria.

11 Recrutant parmi les chiites, on trouve deux groupes majeurs : le mouvement Amal, qui regroupe 5000 à 6000 combattants, et qui avait été créé au début du conflit par le chef religieux de la communauté, l'imam Moussa Sadr. Puis, à partir de 1982-1983, sous l'impulsion de la révolution iranienne et d'un certain nombre de cadres venus d'Iran, le Hezbollah, qui aujourd'hui peut compter sur 3000 à 4000 combattants. Deux groupes aux relations très antagonistes et qui, depuis 1988, ont aussi eu entre eux des affrontements très meurtriers.

Enfin, dans la communauté druze, le Parti socialiste progressiste de Walid Joumblatt qui regroupe environ 4000 à 5000 combattants.

13 Si nous prenons cette population milicienne comme un ensemble, nous pouvons faire quelques remarques sur sa composition sociale. D'abord, dans ce groupe de 30000 miliciens, la répartition par confession est la suivante : $45 \%$ d'entre eux sont des chrétiens, $32 \%$ des chiites, $20 \%$ des Druzes, $2 \%$ des sunnites et $2 \%$ des alouites. Remarquons que les Druzes sont sur-représentés par rapport à leur poids dans la population globale libanaise, et les sunnites sont sous-représentés. En gros, nous pouvons dire que $85 \%$ des miliciens viennent des trois principales communautés d'origine rurale : les maronites, les chiites, les Druzes.

Deuxième remarque: que représente cette population de miliciens par rapport à l'ensemble de la population? Si nous incluons non seulement les 30000 miliciens actuellement dans les différents groupes, mais aussi tous ceux qui ont appartenu à une milice à un moment ou un autre, pour une période de temps significative, nous pouvons estimer qu'il s'agit d'un groupe de 10000 personnes. Ce chiffre représente près de $15 \%$ de la population masculine libanaise âgée de quinze ans et plus. C'est à dire qu'une personne sur six de ce groupe d'âge a, à un moment ou à un autre, effectivement participé, les armes à la main, à la guerre interne. Ce qui nous montre à la fois l'importance et les limites de ce groupe au sein de la société libanaise dans son ensemble. Et si nous revenons au chiffre actuel de 30 000, il s'agit à peine de $5 \%$ de la population active masculine libanaise. 
15 Troisième remarque: il est évident que la composition sociale de la population milicienne a changé, parce qu'il s'agit d'un conflit prolongé qui a connu un cycle de vie complexe. Au départ, les cohortes des années 1975-1976 se composaient d'un mélange hétérogène de chefs de quartier et $d$ 'hommes forts traditionnels (les qabadayes et leurs hommes), de groupes d'autodéfense locaux dans les quartiers et les villages, souvent organisés d'une manière ad hoc, de militants d'un certain nombre de partis d'avantguerre, de gauche ou de droite, engagés dans les combats sous différentes bannières et idéologies, et de jeunes gens des classes moyennes qui s'engagèrent aussi dans le conflit, pleins d'idéaux et d'illusions. A partir du milieu des années 80 , à travers tout un processus de changement, de désengagements et de mobilité sociale forcée, nous arrivons à un tout autre profil social. Il s'agit essentiellement maintenant de milliers de tout jeunes gens - l'âge moyen est tombé très nettement : de 23-25 ans à 15-18 ans. Ces très jeunes sont quasi illettrés, et, pour la plupart, n'ont jamais connu d'autre emploi ; la milice est leur première activité en dehors du cercle domestique. Souvent, ce sont des fils de familles déplacées par les différents aléas du conflit, d'une région à une autre, d'un village à un autre, avec la formation de différents ghettos communautaires. Très souvent aussi, ce sont des consommateurs de drogues et de toutes sortes d'autres excitants. Bref, nous sommes en présence d'enfants de la guerre qui ri ont connu que ce contexte, et qui ont très peu de conception de ce qu'était le Liban d'avant 1975.

Nous avons eu aussi un changement de type socio-géographique. Au départ, la plupart des combattants était de jeunes recrues provenant des quartiers populaires et des banlieues des grandes villes du Liban au milieu des années 70 (les banlieues populaires de Beyrouth, Tripoli, Saïda, et Zahlé). Ces jeunes étaient souvent des fils d'immigrants d'origine plus ou moins récente. Mais, plus tard, à partir du milieu des années 80 , nous avons un afflux de combattants recrutés essentiellement dans les petites villes et les villages des périphéries rurales du pays. Il s'agit pour les maronites des périphéries du nord, beaucoup plus pauvres, plus démunies - des districts de Batroun, de Bécharé, de Akkar, par exemple. Pour les chiites, il s'agit des zones les plus déshéritées du Sud entre Zahrani et le Litani - ou de certaines régions nord de la Bekaa. Pour les Druzes, il s'agit du haut Chouf ou du Metn, qui sont, par rapport à d'autres régions druzes, les plus pauvres et les plus marginales.

17 Dans le milieu des années 80, deux insurrections de milices symbolisent un peu ce passage d'une composition socio-géographique à l'autre. En février 1984, BeyrouthOuest tombe sous le contrôle des milices de Amal et du PSP, et, en mars 1985, BeyrouthEst tombe sous le contrôle des Forces libanaises de Samir Geagea, en révolte contre la direction du parti phalangiste dominée par le président Amine Gemayel. Dans les deux cas, la population urbaine a perçu ce mouvement politico-militaire comme une sorte de prise de contrôle par les régions périphériques, comme une forme $\mathrm{d}$ "'invasion" de la ville par les combattants sudistes chiites à Beyrouth-Ouest et nordistes maronites à Beyrouth-Est.

Anatomie d'une contre-société

Il a fallu évidemment plusieurs années, et plusieurs stades, pour que se consolide ce système de guerre, et qu'il se transforme en une sorte de contre-société qui en est venue à dominer complètement la scène libanaise, la société civile et l'État. Cette contre-société, une fois qu'elle a'est consolidée, comportait évidemment différentes strates sociales. Au sommet, nous avons un groupe limité de "seigneurs de guerre" 
alliés avec les profiteurs de guerre, qui ont fait fortune à travers les différents trafics, extorsions et profits liés au conflit.

Au-dessous d'eux s'est cristallisée une strate de classes pseudo-professionnelles qui ont peuplé les administrations parallèles que les milices ont tenté d'édifier dans les différentes enclaves confessionnelles. Tout un groupe de pseudo-administrateurs, de cadres de milices qui se sont proclamés officiers, idéologues et hommes de médias (les différents radios, télévisions, publications, etc.), d'employés des services logistiques, des services sociaux, des ports illégaux, des réseaux de levée des impôts et des différentes douanes internes, etc.

Plus bas, et liée à ce système, toute une strate parasite d'intermédiaires, de transitaires, de transporteurs, qui ont vécu et se sont développés en s'appuyant sur la fragmentation de l'espace politique et économique que le système milicien a imposée au pays et aussi en profitant de tous les échanges de population, les échanges de propriétés et les nouveaux circuits commerciaux qui ont été créés par les différents déplacements forcés entraînés par la guerre. Plus bas encore, nous trouvons évidemment la base du pouvoir de ce système, c'est-à-dire les combattants, les 30000 combattants à temps plein ou partiel, qui ont profité avec leurs familles d'un certain nombre d'avantages sociaux, d'accès privilégiés à certains biens parfois rares comme l'essence, l'eau, l'électricité, et qui ont bénéficié, dans une certaine mesure, des fruits du pillage de l'économie civile par les seigneurs de la guerre.

21 Enfin, au bas de l'échelle, nous trouvons les segments les plus pauvres, les segments qui proviennent de la population qui n'a pas pu se réinstaller. Cette population déplacée représente un poids considérable puisque, à partir du milieu des années 80 , on peut considérer que plus de $40 \%$ de la population résidente du Liban avait quitté ses régions d'origine à un moment ou à un autre de la guerre. Donc, il s'agit d'une base potentiellement très large. Cette population déplacée a servi de masse de manoeuvre, de base de recrutement, pour les seigneurs de guerre, qui ont tenté de la contrôler en y recrutant des combattants, en y distribuant - parcimonieusement - des biens et des services de toutes sortes, et en y diffusant une idéologie faite de promesses de retour, de revanche, de reconquête. En termes de poids social, si nous incluons les combattants, les employés civils de l'appareil milicien, les profiteurs et les petits intermédiaires liés au système de guerre, il s'agit en gros d'une masse représentant peut-être $20 \%$, au maximum $25 \%$, de la population libanaise.

L'émergence d'une économie noire

22 Ce système de guerre, cette contre-société, a généré progressivement une base économique et institutionnelle qui lui a permis de contrôler progressivement, et de dominer à la fin, les circuits économiques libanais, et de créer ses propres institutions autonomes. À la fin des années 80, un certain nombre de ces milices contrôle des télévisions, des radios, des ports privés, des petits systèmes de transport public, des petits systèmes de sécurité sociale, des dispensaires, etc. Tout cela repose sur le fait que, depuis 1975, l'émergence de ce système de guerre a été accompagnée par la croissance graduelle d'une économie parallèle ou d'une économie noire. Quelles ont été les sources de cette économie, les bases matérielles de ce système milicien? Rapidement, nous pouvons dire qu'elles sont les suivantes :

D'abord, il y a eu la destruction et le pillage des avoirs et stocks de la vieille bourgeoisie, de la bourgeoisie traditionnelle de l'avant-guerre. En 1975-1976, dans la première phase de la guerre, le centre de Beyrouth, avec ses milliers de magasins, avec ses banques, ses 
entrepôts, et le port de Beyrouth ont été systématiquement pillés par les différentes milices à Beyrouth-Est et à Beyrouth-Ouest, et souvent à travers un système de partage plus ou moins à l'amiable des zones de pillage. On estime à peu près à 800 millions de dollars la valeur des biens qui ont été pillés au cours des deux premières années du conflit. En 1975-1976 a commencé aussi, à une moindre échelle, le pillage du secteur Hamra Ras-Beyrouth, qui était le deuxième centre commercial du Grand Beyrouth. Ce secteur a été beaucoup plus affecté dans la période 1984-1986, après la faillite de la reconstruction de l'autorité centrale sous Amine Gemayel. De même, dans les environs de Zahlé et de Saïda, les établissements agricoles et commerciaux ont commencé à être pillés dès 1976. Bref le pillage de la bourgeoisie traditionnelle et de ses centres commerciaux et financiers a été la première source de l'accumulation des sources de revenus des milices.

Une deuxième source a été la saisie, le pillage, et souvent la vente forcée de propriétés des membres d'une certaine communauté qui avaient quitté ou qui se préparaient à quitter un territoire contrôlé par les milices d'une autre communauté - les chrétiens à Beyrouth-Ouest, les musulmans à Beyrouth-Est, etc. Les milliers de propriétés ont changé de main, très souvent pour des sommes tout à fait dérisoires, et ont été en partie récupérées ou fortement taxées par les différents appareils miliciens.

La troisième source de revenus, c'est la taxation illégale de la population résidente dans l'enclave contrôlée par telle ou telle milice. Les différentes milices ont levé des taxes municipales, elles ont pris le contrôle des taxes sur les transferts de propriété, elles ont imposé des taxes indirectes sur l'essence, les cigarettes, les restaurants, les activités de loisir, etc., et parfois ces taxes apparaissent dans les factures que les marchands ou que les fournisseurs de services remettent à leurs clients.

La quatrième source de revenus, c'est le racket au sens traditionnel, l'extorsion d'argent contre la protection des hommes d'affaires et des industriels. Cette extorsion a pris soit la forme de versements forfaitaires par entreprise ou par usine, soit celle d'imposer des combattants de telle ou telle milice comme gardes du corps, comme gardiens, ou le plus souvent dans tel ou tel emploi fictif L'entreprise les paie comme salariés, ce qui revient à faire payer par ces entreprises le salaire d'un certain nombre de miliciens.

La cinquième source a été l'installation de douanes internes d'un territoire à l'autre douanes terrestres, sur les points de passage entre les différentes enclaves, douanes maritimes dans un certain nombre de ports légaux que telle ou telle milice contrôlait (Beyrouth, Tripoli, Saïda, Jounieh), ou de petits ports illégaux qui ont proliféré sur la côte libanaise.

Pour illustrer la croissance de ces douanes illégales imposées par les milices, je donnerais ici simplement l'exemple des revenus douaniers de l'État, qui ont diminué évidemment en proportion inverse de la croissance de cette économie milicienne. En 1980, l'État touchait encore $90 \%$ des revenus de douanes estimés ; en 1983, sa part était tombée à $60 \%$ des revenus estimés ; et, en 1986, à $10 \%$, c est dire que pratiquement, en cinq ou six ans, les revenus douaniers de l'État ont complètement disparu au profit des milices. Enfin, une sixième source très importante de revenus de l'économie milicienne a été la contrebande - la contrebande de drogue, d'armes et de biens volés. Des biens volés à l'intérieur d'une région et revendus dans une autre, mais aussi des biens volés à l'extérieur à travers des actes de piraterie maritime ou de détournements de navires marchands vers les ports illégaux libanais. Pour donner un exemple de la croissance de 
ce type de contrebande, je citerai un seul chiffre : la production de drogue à l'intérieur du territoire libanais s'est élevée de 500 millions de dollars en 1981 et à 1200 millions de dollars en 1988. On estime que le système milicien a prélevé au moins 20 à $30 \%$ de la valeur de cette somme comme profit au passage.

Jusqu'en 1982, cette économie noire ou parallèle était encore relativement contenue et ne représentait peut-être pas plus de $20 \%$ de l'économie globale du Liban. Mais à partir du milieu des années 80, après l'invasion israélienne de 1982, l'écroulement de la tentative de reconstituer l'État en 1982-1983, et la segmentation encore plus grande de l'espace économique et politique, on estime aujourd'hui que l'économie noire représente plus de $50 \%$ de l'ensemble de l'économie libanaise. Dans la dernière phase de cette évolution de l'économie milicienne, il y a eu toute une série de développements nouveaux : les milices se sont mises à créer un nombre de sociétés de holding en association avec des profiteurs de guerre et même des éléments de la bourgeoisie classique dans le but de diversifier leurs activités, et de reconvertir un certain nombre de leurs revenus en revenus "réguliers". Au cours des années 19851990 , sont nées toute une série d'entreprises de multimédias, de régies de publicité, de sociétés de computer et de software, de sociétés de commerce extérieur, de sociétés d'investissement et même de sociétés d'investissement sur le marché international.

Les règles du jeu milicien

31 Le quatrième aspect que je voudrais examiner brièvement dans cette anatomie du système de guerre au Liban, ce sont les règles de fonctionnement de ce système. Une analyse comparée des différentes phases du conflit et des différents changements permet de dégager un certain nombre de régularités qui éclairent le fonctionnement de ce système jusqu'à aujourd'hui.

Première règle : la primauté du conflit intra-communautaire. Les différentes milices ont essayé de détruire à l'intérieur de leur propre communauté la vie politique pluraliste qui existait auparavant, et ont essayé de monopoliser le pouvoir politicomilitaire. Au sein de la communauté druze, le succès a été très large, sinon total. La fraction traditionnelle des Yazbeki, qui partageait le pouvoir et l'inf1uence avec les Joumblattis, a nettement décliné et a été réduite à une position tout à fait subordonnée: pour la première fois dans l'histoire contemporaine des Druzes, nous avons un système unipolaire. Chez les maronites, le succès a été partiel. Les forces libanaises sont arrivées à marginaliser et à réduire l'influence de beaucoup de partis politiques, de factions - y compris le parti dont elles sont elles-mêmes originaires, le parti phalangiste, et aussi le parti national libéral de Camille Chamoun et le bloc national de Raymond Eddé. Elles ont aussi marginalisé les personnalités indépendantes; mais elles n'ont pas pu empêcher une scission du Nord, essentiellement sous la direction du clan des Frangié et de la milice des Maradas, et n'ont pas pu arrêter un processus de scission interne qui les a affecté elles-mêmes. Nous avons vu le parti phalangiste se diviser, les forces libanaises se fractionner, et, dans la dernière période, les forces libanaises et les brigades à majorité chrétienne de l'armée libanaise s'affronter à l'intérieur des zones de Beyrouth-Est de façon tout à fait sanglante. Donc, on peut parler de succès partiel jusqu'à il y a deux ou trois ans et d'échec depuis. Au niveau de la communauté chiite, cette tentative de domination d'un groupe n'a pas réussi, et il s'est installé une forte bipolarisation instable et souvent sanglante entre les deux factions de l'Amal et du Hezbollah. Quant aux sunnites, ils ont connu une fragmentation de plus en plus grande et une dépendance dans les 
différentes régions où ils habitent par rapport aux milices des autres communautés. Aucune des petites factions sunnites qui ont émergé pendant le conflit n'est arrivée à avoir une puissance et une influence suffisante pour être autonome ou avoir un poids significatif dans le conflit. Mais je rappelle ici qu'essentiellement cette règle du jeu veut dire que la plupart des batailles significatives - celles qui ont vu le plus grand nombre de victimes parmi les miliciens eux-mêmes, et des tentatives véritables d'offensive, d'avance, etc.-, ce sont des batailles qui se sont déroulées à l'intérieur des communautés et pour le contrôle politico-militaire de la communauté et de son territoire.

33 Ce qui nous amène à la deuxième règle du jeu: il y a eu effectivement des combats inter-communautaires, mais jamais vraiment pour obtenir une victoire. Au cours du conflit, il y a eu très peu de tentatives véritables de vaincre décisivement les autres factions ou d'envahir leurs territoires ou leurs sanctuaires. Les confrontations majeures inter-communautaires se sont déroulées sur un certain nombre de lignes de démarcation qui ont très peu changé en quinze ans, et ont eu lieu, soit à travers des bombardements mutuels aveugles et à distance, soit à travers l'emploi de voitures piégées qui explosaient au milieu des populations civiles. La fonction essentielle de ce combat inter-communautaire n'était ni la victoire, ni le contrôle de l'État, ni le contrôle du territoire de l'autre; c'était de légitimer la prétention des milices d'être les défenseurs de leur propre communauté et de leur propre territoire en agitant la crainte des autres, le bombardement des autres, le danger d'invasion des autres. Donc, il s'agissait essentiellement d'une fonction négative et auto-justificatrice.

La troisième règle du jeu de ce système milicien, c'est le changement quasi illimité des alliances. Aucune combinaison n'est vraiment impensable; presque toutes les possibilités ont été essayées. C'est un système kaléidoscopique de composition et de décomposition des alliances, et nous pouvons donner toute une série d'illustrations d'anciens ennemis qui sont devenus alliés à un certain moment. L'exemple le plus spectaculaire est peut-être celui des forces libanaises et de l'OLP, qui étaient les protagonistes majeurs du conflit en 1975-1976 et qui, à travers une série de changements, se retrouvent dans une posture d'alliés indirects et parfois même directs, à partir de la guerre des camps en 1985-1986, en fonction de leur opposition mutuelle à la Syrie. La même chose peut être dite d'un certain nombre de mouvements islamistes de Tripoli, d'un certain nombre de factions chiites de Beyrouth-Ouest, etc. La quatrième règle du jeu, c'est la perméabilité relative des factions. Tout au long du conflit, des dirigeants d'un groupe ou d'une faction sont, après leur défaite, passés dans le territoire de la faction à laquelle ils étaient théoriquement opposés. Lorsque les cadres chamounistes ont été défaits par Bechir Gemayel et les combattants phalangistes en 1980-1981, un certain nombre d'entre eux ont quitté Beyrouth-Est pour BeyrouthOuest. lorsque Hobeika et ses associés ont été défaits par Geagea en 1985, ils sont passés à Beyrouth-Ouest. Lorsque les forces syriennes et leurs alliés sont entrés à Tripoli en 1985-1986, un certain nombre de dirigeants, y compris ceux des mouvements intégristes sunnites de Tripoli, sont passés à Beyrouth-Est. Et nous avons évidemment à l'intérieur même de chacune des régions et des communautés un passage continu de combattants et de commandants d'une faction à une autre. Une bonne partie des conflits entre Amal et Hezbollah en 1987-1989 ont vu des cadres et des combattants passer d'une faction à une autre, souvent à la suite "d'encouragements" financiers de toutes sortes. 
d des arrangements entre les différentes milices pour l'essence et les produits énergétiques, il y en a eu pour la distribution du tabac local importé. Les circuits de la drogue ont fonctionné au cours des différentes phases du conflit sans aucun problème, et ont traversé toutes les enclaves et toutes les frontières. Les marchandises et les voitures ont circulé et circulent d'une région à l'autre ; très souvent les marchandises et les voitures volées à Beyrouth-Ouest sont revendues à Beyrouth-Est, et vice versa. De nombreux témoignages ont montré que la solidarité fiscale fonctionnait très bien. Les marchands de Beyrouth-Est, qui devaient payer leur dû aux milices de Beyrouth-Ouest s'ils voulaient vendre à Beyrouth-Ouest, se voyaient réclamer les arriérés par les milices de Beyrouth-Est: la réciproque étant de rigueur. Certaines des milices de Beyrouth-Ouest venaient même chez les marchands de Beyrouth-Est leur dire : "Vous devez telle ou telle somme aux forces libanaises, et vous allez le payer". Ainsi, la solidarité des miliciens contre les marchands était une des règles les mieux respectées du système.

Sixième règle : à plusieurs reprises, les milices ont tenté d'établir un système global de partage du pouvoir entre elles, un système qui irait au-delà de l'économie de guerre, qui essayerait de la consolider à travers une nouvelle structure politique. L'exemple le plus spectaculaire est évidemment l'accord tripartite de 1985 entre les trois principales milices, mais beaucoup de tentatives précédentes (en 1976, en 1984)) et les tractations politiques de cet automne 1990 comportent des éléments de cette tentative de transformer cette association des milices en partie intégrante de la structure politique officielle.

Enfin, septième et dernière règle du jeu : c'est la défense commune du système de guerre, surtout contre les tentatives de reconstruire un État central et de reconstituer une armée nationale, chacun résistant dans son propre territoire et souvent arguant de l'attitude et des positions des autres pour bloquer ou pour saboter ces tentatives. Chacune des milices prétendait évidemment qu'elle accepterait le retour de l'État lorsque les autres l'accepteraient et que l'autorité de l'État ou de l'armée devait d'abord être imposée aux autres et ensuite à elle-même. Il y a également eu une convergence et une complicité des milices dans la répression de la société civile, la répression des individus et des groupes qui ont résisté à l'imposition de ce système de guerre. A partir du milieu des années 80 , la société civile a commencé à réagir à la banqueroute économique et à la crise sociale, à la désintégration du pays, à travers de très grandes manifestations, des grèves, des sit-in, et toutes sortes de protestations sociales. Le système milicien a réagi en menaçant les syndicats, en menaçant les associations professionnelles, les administrations de l'État, en kidnappant un certain nombre de leaders syndicaux et professionnels, en sabotant des réunions et des manifestations de masse, en harcelant la partie de la population qui continuait à se déplacer entre les différents territoires, en créant toute une série de points de contrôle où ceux qui traversaient étaient kidnappés, humiliés, surveillés, etc., en limitant la consommation et la circulation des livres et de la presse d'un territoire à l'autre. Bref, en réprimant toutes les tentatives de la société civile de résister et de combattre le système de guerre.

Vers un démantèlement du système de guerre? 
39 Aujourd'hui, à l'automne 1990, nous assistons de nouveau à une tentative de démanteler le système milicien et de reconstituer, dans des conditions difficiles, l'autorité de l'État et une forme de réconciliation politique. Le système milicien va tenter de nouveau de saboter cette entreprise. Peut-être que, dans la conjoncture actuelle, il sera obligé de faire un certain nombre de concessions. Ces concessions, essentiellement, consisteront, comme cela s est passé déjà à plusieurs reprises, dans un retrait hors du Grand Beyrouth de leur effectifs professionnels militaires, de leurs armements lourds et de certaines de leurs infrastructures vers des sanctuaires dans le mont Liban pour les forces libanaises (le Kesrouan ou Jbeil), vers le Chouf pour le PSP, vers le Liban-Sud pour Amal, vers la Bekaa pour le Hezbollah. Ce retrait visible, s'il se fait, masque d'autres stratégies. Soit celle de pouvoir pénétrer directement l'appareil central d'État à travers la nomination d'un certain nombre de dirigeants de ces milices comme députés ou comme ministres, soit à un stade ultérieur, quelques mois plus tard, et lorsque la conjoncture le permettra, celle de se ré-infiltrer dans le Grand Beyrouth et de saboter de l'intérieur cette base territoriale à partir de laquelle l'état essaie de se reconstituer et de mettre fin à ce système de guerre. C'est pour cela qu'il est très important non seulement d'obtenir des concessions des milices au niveau de leur retrait géographique du Grand Beyrouth, mais surtout de s'atteler à démanteler les bases économiques, sociales et politiques de ce système de guerre. Il s'agit de s'atteler à un plan à long terme de résorption de cette économie de guerre, de résorption de ses combattants, de solution au problème des populations déplacées, de réactivation d'un processus politique démocratique qui permettra à la population dans ses différentes composantes de s'exprimer, de se faire représenter, de constituer un nouveau leadership politique légitime capable de couper définitivement l'herbe sous les pieds de toute cette caste, dont le pouvoir économique, militaire et politique s'est constitué sur les dépouilles de la société civile libanaise, de l'économie civile libanaise, et de l'État libanais. Le problème du démantèlement d'un système qui a mis plus de quinze ans à se former et à se consolider et qui a pris des racines institutionnelles, économiques et sociales profondes dans le territoire libanais n'est pas un problème simple, il ne peut être résolu uniquement par des décrets et des négociations. C'est un long processus de destruction et de résorption de ce qui a été le produit d'un conflit complexe et prolongé. C'est aussi un processus de reconstruction d'une société démocratique, civile et pluraliste.

\section{RÉSUMÉS}

Au lieu de se pencher sur les origines du conflit et d'en tirer souvent l'idée d'une prédisposition à la guerre des communautés libanaises alimentée par l'étranger, il est nécessaire d'étudier la dynamique du processus conflictuel lui-même. Ce processus a fait émerger un " système de guerre " qui a ses propres acteurs et se structure comme une contre-société. Il se prolonge grâce au contrôle d'une économie noire qui est faite de trafics (douanes illégales, taxations, drogue...) et qui finit par créer entre les acteurs militarisés des intérêts objectifs à la perpétuation du 
conflit au détriment de la société civile libanaise. Il n'est pas sûr malgré les événements récents que nous allions vers le démantèlement de ce système de guerre.

Instead of studying the origins of the conflict or thinking of a liability to develop a war of the Lebanese communities and the regional powers, it is preferable to study the dynamic of the conflict process itself. This process has brought the possibility for the emergence of a " war system " with its own actors and is built as a counter-society It is extended because of the control of a black economy made of traffics (illegal customs, taxation, drugs...) and creates amongst military actors some objective interests for the perpetuation of the conflict. This perpetuation proved detrimental to the Lebanese civil society and the latest events do not permit foreseeing a dismantling of this type of " war system ".

\section{INDEX}

Mots-clés : conflits, guerre

Index géographique : Liban

\section{AUTEUR}

\section{SALIM NASR}

Sociologue, Professeur à l'Université de Georgetown (USA), département Monde Arabe. 